\title{
Current status of uterine regenerative medicine for absolute uterine factor infertility (Review)
}

\author{
YUSUKE MATOBA, IORI KISU, ASAKO SERA, MEGUMI YANOKURA, KOUJI BANNO and DAISUKE AOKI \\ Department of Obstetrics and Gynecology, Keio University School of Medicine, Tokyo 160-8582, Japan
}

Received July 16, 2018; Accepted November 16, 2018

DOI: $10.3892 /$ br.2019.1182

\begin{abstract}
Though assisted reproduction technology has been developed, a treatment for absolute uterine factor infertility (AUFI), such as defects in the uterus, has not yet been established. Regenerative medicine has been developed and applied clinically over recent years; however, whole solid organs still cannot be produced. Though uterine regeneration has the potential to be a treatment for AUFI, there have been only a few studies on uterine regeneration involving the myometrium in vivo. In the present report, those relevant articles are reviewed. A literature search was conducted in PubMed with a combination of key words, and 10 articles were found, including nine in rat models and one in a mouse model. Of these studies, eight used scaffolds and two were performed without scaffolds. In four of these studies, scaffolds were re-cellularized with various cells. In the remaining four studies, scaffolds were transplanted alone, or other structures were used. Though the methods differed, the injured uterus recovered well, morphologically and functionally, in every study. Only 10 articles were relevant to our investigation, but the results were favorable, if limited to partial regeneration. Recently, uterus transplantation (UTx) has been investigated as a treatment for AUFI. However, UTx has many problems in the medical, ethical and social fields. Though the artificial uterus was also researched and some improvements in this technology were reported, it will take long time for this to reach a clinically applicable stage. Though the results of uterine regeneration studies were promising, these studies were conducted using animal models, so further human studies and trials are needed.
\end{abstract}

Correspondence to: Dr Iori Kisu, Department of Obstetrics and Gynecology, Keio University School of Medicine, 35 Shinanomachi, Shinjuku-ku, Tokyo 160-8582, Japan

E-mail: iori71march@hotmail.co.jp

Key words: absolute uterine factor infertility, bioengineering, uterine regeneration, tissue engineering, Mayer-Rokitansky-Küster-Hauser syndrome

\section{Contents}

1. Introduction

2. Methods

3. Results

4. Discussion

5. Conclusion

\section{Introduction}

The World Health Organization defines infertility as a disease resulting in a failure to conceive after at least one year of unprotected intercourse (1). Recent developments in assisted reproduction technology have promoted treatment options and success for many infertile patients. However, a specific treatment for absolute uterine factor infertility (AUFI) has not yet been established. AUFI is defined as an infertile condition caused by problems with the uterus, and is classified as either congenital or acquired. Congenital AUFI can include uterine malformation such as Mayer-Rokitansky-Küster-Hauser syndrome, which occurs in one in 4,500 female children (2). Acquired AUFI is usually caused by hysterectomy for a malignant uterine tumor, including cervical and endometrial cancer, benign diseases such as uterine fibroids, and postpartum hemorrhage (3). Asherman syndrome and severe endometrial adhesion are also causes of AUFI.

Surrogate pregnancy and adoption are both options by which patients with AUFI may have their own biological child. However, more countries are prohibiting surrogacy because of the medical risks to surrogate mothers and concerns about the welfare of the child, causing difficulties and increased mental stress for patients with AUFI. Thus, research on uterine regenerative medicine was initiated as a solution to AUFI. Organ regeneration was established in the 1970s, based on the culture of differentiated cells, such as epidermal cells and chondrocytes (4). Langer and Vacanti (5) showed that the use of scaffolds allowed cells to settle in transplanted sites more effectively than with injecting suspensions locally. This result led to the concept of tissue engineering (6). Nowadays, multipotent cells, such as embryonic stem cells (ESCs) and induced pluripotent stem cells, are used for studies in this field $(7,8)$. Furthermore, scaffolds that can more accurately recapitulate the complexity of vascular structures and those produced by the de-cellularization of organs are used in 
tissue engineering research (5). Skin (9), cartilage (10), blood vessel (11), liver (12), heart (13) and kidney (14) regeneration has been studied; skin and cartilage regeneration has been used in clinical practice $(15,16)$. However, whole solid organs still cannot be produced and various devices for this purpose are currently under development (17).

Though it is important to identify a specific treatment for patients with AUFI to relieve the mental burden of this condition and allow them to have their own biological child, there have been only a few studies thus far on uterine regeneration as a whole organ. In the present report, studies on uterine regeneration containing the myometrium in vivo have been reviewed, in order to present and discuss the current status of uterine regeneration technology, which has potential to successfully treat patients with AUFI.

\section{Methods}

A literature search in PubMed was conducted using the combination of key words 'uterus', 'uterine', 'engineering', 'reconstruction' and 'regeneration' to collect articles relevant to uterine regeneration. These were examined, though only data from those that referred to uterine regeneration involving the myometrium in vivo were collected. No specifications on animal species were placed. A manual search of the bibliographies of relevant papers was carried out to identify additional studies for possible inclusion. Articles written in a language other than English were excluded from the present review.

\section{Results}

A total of 10 articles were found, including nine involving rat models and one with a mouse model. Of these, eight studies used scaffolds and two were performed without scaffolds (Table I).

Partial regeneration of the uterus using scaffolds. Li et al (18) transplanted collagen scaffolds with collagen-binding domain attached to the N-terminal of native basic fibroblast growth factor (CBD-bFGF) and scaffolds with native basic fibroblast growth factor (NAT-bFGF) in rats. Three groups were compared: Transplant scaffolds with CBD-bFGF (CBD-bFGF group), transplant scaffolds with NAT-bFGF (NAT-bFGF group), and a non-transplant group. Patent uterine cavities accounted for $45 \%$ in the non-transplant group, $85 \%$ in the NAT-bFGF group, and $95 \%$ in the CBD-bFGF group. Endometrial thickness, number of neovessels, regeneration of myocytes, and cell proliferation (assessed by Ki-67 immunostaining) were each highest in the CBD-bFGF group. A pregnancy test showed pregnancy in the transplanted uteri, with rates of $86.67 \%$ in the CBD-bFGF group, $60.00 \%$ in the NAT-bFGF group, and $33.33 \%$ in the non-transplant group. Based on these results, it was concluded that a bFGF delivery system using a collagen scaffold may be useful for uterine regeneration.

Santoso et al (19) cut the uterine horns of Sprague Dawley rats into $15 \times 5 \mathrm{~mm}$ rectangular sections and de-cellularized the samples with ionic detergent, SDS or high hydrostatic pressure (HHP) to produce scaffolds. A defect of approximately $15 \times 5 \mathrm{~mm}$ was created, and the scaffold was transplanted into recipient rats. At 30 days after transplantation, changes in the uteri of groups transplanted with a scaffold prepared using SDS (SDS group) or HHP (HHP group) were compared with those in a sham group, in which the uterus was only incised and sutured, and a native group, in which the uterus had no intervention. The transplants in the SDS and HHP groups were found to have macroscopically assimilated with the surrounding tissues by 30 days post-transplantation. Histology revealed regenerated epithelial and stromal cells and myocytes in both scaffold-transplant groups. The increases in elastin and collagen in the uteri of both scaffold-transplant groups were similar to those of the sham group. In a comparison of mechanical properties, there were no significant differences in Young's modulus and tissue thickness between the scaffold-transplant and sham groups. However, significantly more fatty tissues were attached to the transplant site in both the scaffold-transplant groups and to the surgical site in the sham group, in comparison with the native group. This may influence the mechanical properties of these groups. In subsequent pregnancy tests, there was no significant difference in the number of fetuses among the four groups. These results suggest that scaffolds produced using SDS or HHP methods contribute to uterine tissue regeneration both morphologically and functionally.

Miyazaki and Maruyama (20) also achieved partial regeneration of the uterus in rats. The uterus, including the abdominal aorta, was removed from each rat and perfused with SDS from the aorta for de-cellularization. A mixture of cells extracted from a rat uterus and rat mesenchymal stem cells (MSCs) were injected to induce re-cellularization, and then the re-cellularized scaffold was transplanted into a rat uterine partial defect injury model. Rats were divided into three groups: A transplant group, in which the graft was transplanted into the uterine defect, a non-transplant group, in which only a uterine defect was made, and a control group, in which no procedure was performed. Regenerated tissues in the transplant group were thicker than those in the non-transplant group. Immunohistochemistry (IHC) showed vimentin-positive cells in the transplant sites and regeneration of stromal cells, and confirmed that cytokeratin-positive cells covered the lumen, indicating epithelialization. In a pregnancy test, pregnancy was confirmed in one of six uterine horns in the non-transplant group, and six of eight uterine horns in the transplant group. However, there were significantly fewer fetuses in the transplant and non-transplant groups, compared with the control group. The function of regenerated uteri with endometrial decidualization, which is identified by desmin-positive cells in rats using immunostaining, was also assessed. In the transplant group, desmin-positive cells increased similarly to the control group and hormone sensitivity was confirmed, whereas these changes were not found in the non-transplant group. Based on these results, it was suggested that the regenerated endometria exhibited both morphological and functional regeneration.

Ding et al (21) reported on the re-cellularization of bone marrow (BM)-derived MSCs in collagen scaffolds and transplantation into rats. In this study, rats were divided into four groups: An MSC/scaffold group, in which collagen scaffolds with MSCs were transplanted into the uterine defect; a scaffold-transplant group, in which scaffolds without MSCs were transplanted; a non-transplant group, in which a uterine defect 


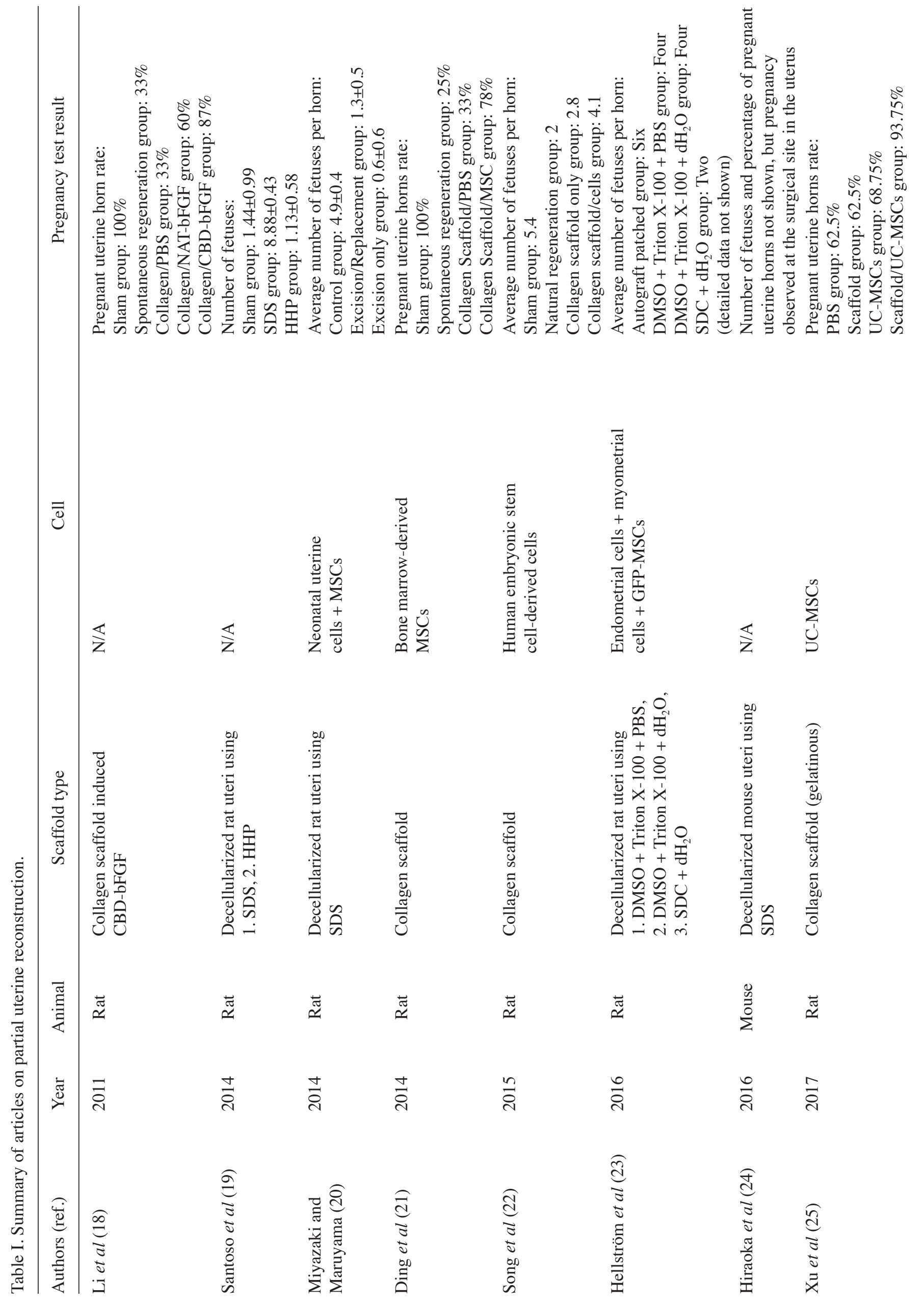


was made without a transplant; and a sham group, in which no procedure was performed. In the MSC/scaffold group, expression of various markers, including bFGF, IGF-1, TGF $\beta 1$ and VEGF, was higher than that in the scaffold-transplant group and the non-transplant group. Proliferation of endometrial cells and myocytes and vascular density in the MSC/scaffold group were also higher than those in the scaffold-transplant and non-transplant groups. A pregnancy test on day 90 after transplantation confirmed pregnancy in 6/18 transplanted uterine horns in the MSC/scaffold group, which was inferior to $100 \%$ in the sham group, but superior to the results in the scaffold-transplant and non-transplant groups. Based on these results, Ding et al (21) suggested that the scaffold with BM-MSCs may contribute to regeneration of uterine tissues.

Song et al (22) also showed regeneration of the uterus in rats. Collagen scaffolds were used and human ESCs were cocultured with endometrial cells for use as ESCs-differentiated cells for cellularization of scaffolds. Four groups were compared: A cellularized scaffold (cells/scaffold) group, scaffold only group, resection only group, and a sham (no procedure) group. The rate of patency of the uterine cavity was high (90\%) in the cells/scaffold group, ranked second to the sham group. In the cells/scaffold group, assimilation with surrounding tissues was enhanced, with increased endometrium thickening, muscle layer thickening, and endometrial glands in comparison with other groups at 12 weeks after transplantation. A pregnancy test showed pregnancy rates of $80 \%$ in the cells/scaffold group, $33 \%$ in the scaffold only group, and $26.7 \%$ in the resection only group. The mean number of fetuses per uterine horn was 4.2 in the cells/scaffold group, which was higher than that in other groups, and implantation around the transplant site was confirmed. Based on these results, Song et al (22) proposed that transplantation of the cellularized scaffold enhances regeneration of uterine function. However, small amounts of transplanted human ESCs-derived cells were detected in the transplant sites, which suggested that transplanted cells did not differentiate similarly to surrounding cells, but enhanced transfer of surrounding cells.

Hellström et al (23) perfused the uterus removed from donor rats using three different methods to produce de-cellularized scaffolds. The three perfusion methods included continuous perfusion from the aorta with perfusate containing $4 \%$ dimethyl sulfoxide (DMSO) and 1\% Triton X-100 in phosphate-buffered saline (PBS) (group 1); perfusion with DMSO and Triton X-100 in distilled water $\left(\mathrm{dH}_{2} \mathrm{O}\right)$ (group 2); and 6-h perfusion with $2 \%$ sodium deoxycholate solution (SDC) in $\mathrm{dH}_{2} \mathrm{O}$ (group 3). Suspensions of a mixture of endometrial and myometrial cells extracted from the rat uterus and green fluorescent protein (GFP) labeled MSCs were injected into the perfused uterus to induce re-cellularization. After re-cellularization of these scaffolds, they transplanted those in a partial defect injury in Sprague-Dawley rat. Grafts of $5 \times 10 \mathrm{~mm}$ were produced and transplanted into recipient rats. A pregnant test was performed and there were no differences in the number of pregnancies between groups 1 and 2, or in the number of fetuses in each uterine horn. However, the number of pregnancies in transplanted uteri was lower in group 3. No placenta was attached to the transplant site in all transplanted groups, but pregnancy around the transplant site was confirmed in groups 1 and 2, whereas no pregnancy was found around the transplant site in 
group 3. Based on these results, Hellström et al (23) suggested that SDC was inappropriate to produce scaffold for partial uterus transplantation and concluded that grafts transplanted in groups 1 and 2 contributed to regeneration of partial defect injury of the uterus. However, GFP-labeled MSCs in the transplanted sites were not detected using IHC and no differentiation of MSCs to uterine tissues was shown. The author mentioned that some beneficial effects may have contributed to a successful repopulation of host uterus cells that eventually replaced the GFP-labeled MSCs in their study.

Hiraoka et al (24) transplanted murine uterus-derived scaffolds that were de-cellularized using SDS into a recipient mouse and confirmed regeneration of epithelial cells and muscle and stromal layers. On day 28 after transplantation, grafts were macroscopically similar to surrounding tissues, and IHC with creatine kinase $8, \alpha$-smooth muscle actin $(\alpha-S M A)$, estrogen receptor and progesterone receptor revealed structures of epithelia, glandular epithelia, and stromal and muscle layers. Cells forming these structures were confirmed to be derived from recipient mice. A pregnancy test showed no significant difference in the number of fetuses in the uterine horns between the transplant and non-transplant groups, with pregnancy at transplanted sites. The body weight of fetuses in the transplant sites was normal. They also performed the experiments about cell migration and hormonal contribution to the regeneration. The experiments revealed that transfer of host epithelial cells to grafts first occurred one day after transplantation, and transfer of cells to the stroma began one week after transplantation. Experiments in ovariectomized mice showed that estrogen and progesterone were not involved in tissue regeneration. However, STAT3, which is involved in cell proliferation in epithelial tissues, was found to be associated with scaffold regeneration in the uterus when using knockdown mice.

$\mathrm{Xu}$ et al (25) injected umbilical cord-derived MSCs (UC-MSCs) and gelatin collagen scaffolds into a scar site in a uterine defect rat model. Four groups were examined: a UC-MSCs/scaffold group, in which UC-MSCs and gelatin collagen scaffolds were injected, a UC-MSCs group, in which only UC-MSCs were injected, a scaffold group, in which only scaffolds were injected, and a PBS group, in which PBS was injected instead of UC-MSCs. In the UC-MSCs/scaffold group, UC-MSCs were retained in the scar site, suggesting a contribution of the gelatin scaffold. Uterine regeneration was found macroscopically in the UC-MSCs/scaffold group and the regenerated conditions 60 days after the operation were similar to those of a normal uterus. Histological examination revealed that uterine regeneration based on endometrial thickness, regeneration of uterine tissues, and the number of endometrial glands and neovessels was greater in the UC-MSCs/scaffold group than in the PBS, scaffold, and UC-MSCs groups. A pregnancy test showed a pregnancy rate of $93.75 \%$ in the UC-MSCs/scaffold group, which was the highest of the four groups. The UC-MSCs/scaffold group had implantation at 10 of 16 uterine horns in scar sites, which was significantly higher than the two in the PBS group, one in the scaffold group, and three in the UC-MSC group. In the molecular biological investigation, high expression of matrix metalloproteinase-9 was found in the UC-MSCs/scaffold group, suggesting that this enzyme might promote regeneration.
Uterine regeneration without scaffolds. Campbell et al (26) generated boiled blood clots with tubular shapes $(0.5 \mathrm{~cm}$ diameter $x 2.5 \mathrm{~cm}$ long), and transplanted them into the abdominal cavity of rats to produce tissue for transplantation. After 2-3 weeks, the clots covered with myofibroblast tissues were removed and carefully detached $(1.5-2.0 \times 0.75-1.0 \mathrm{~cm})$ for transplantation in a uterine defect model in rats. Grafts were assessed at 4, 6, 8, 10 and 12 weeks after transplantation. Morphology of muscle layers, epithelia and secretory glands became similar to those in normal uteri over time. A pregnancy test at 12 weeks after transplantation confirmed pregnancy in all operated rats, with normal fetuses in the transplant sites. Based on these results, Campbell et al (26) concluded that a procedure without scaffolds was also an option to support tissue engineering.

Lin et al (27) showed regeneration of the uterus in a rat partial defect injury model, using injection of native vascular endothelial growth factor (NAT-VEGF) or vascular endothelial growth factor with a collagen binding domain (CBD-VEGF) into the injury site, which was established 30 days prior to injection of NAT-VEGF and CBD-VEGF. In this report, there were three groups: CBD-VEGF injection (CBD-VEGF group), NAT-VEGF injection (NAT-VEGF group), and PBS injection (PBS group). The uterine wall in the CBD-VEGF group was significantly thicker and the uterine glands were significantly better formed than those in the other two groups. IHC showed more $\alpha$-SMA-positive cells in the CBD-VEGF group than in the other groups, suggesting enhanced regeneration of myocytes. IHC with von Willebrand factor revealed a higher density of neovessels in the CBD-VEGF group. A pregnancy test gave pregnancy rates of $56.3 \%$ in the PBS group, $68.6 \%$ in the NAT-VEGF group, and $87.5 \%$ in the CBD-VEGF group, although with no significant differences among the groups. The pregnancy rate at the scar site was significantly higher in the CBD-VEGF group (8/16) compared with the NAT-VEGF $(3 / 16)$ and PBS (1/16) groups. Based on these results, it was suggested that VEGF remained in scar sites due to the CBD and contributed to uterine remodeling.

\section{Discussion}

We have reviewed studies of uterine regeneration, including myometrium regeneration, as a therapeutic approach for AUFI. The studies were roughly classified into those using scaffolds and those without scaffolds. Scaffolds were produced with de-cellularized organs, or collagen scaffolds were used. Scaffolds formed by de-cellularization have a concern of an immune response to grafts, but no studies to date have shown a noteworthy immune response to these scaffolds. Collagen scaffolds are likely to be useful in clinical practice if infection-associated problems can be solved.

It remains unclear which cell strains are best for uterine regeneration. Multipotent BM-MSCs and UC-MSCs and human ESCs have been used; however, no study has shown that these cells differentiate directly into endometrial and myometrial cells. Therefore, there is a need to examine how these cells influence and contribute to uterine remodeling. On the other hand, many studies have shown uterine remodeling via the migration of host cells, and in studies with CBD-VEGF and CBD-bFGF, uterine remodeling was enhanced without 
the transplantation of stem cells. Identification of factors that promote tissue regeneration may lead to the development of new fields of regenerative medicine.

There are few studies on regeneration of all uterine layers, including muscle layers, but regeneration of endometria alone has been examined using several cell types. Gil-Sanchis et al (28) examined endometrial regeneration after injection of cell suspensions of unfractionated bone marrow cells, hematopoietic progenitor cells, endothelial progenitor cells, and MSCs isolated from murine bone marrow-derived cells, and three established cell lines (bone marrow-derived multipotent adult progenitor cells, bone marrow-derived hypoblast-like stem cells, and MSCs). The isolated murine MSCs, endothelial progenitor cells, and bone marrow derived hypoblast-like stem cells contributed to endometrial regeneration. Gan et al (29) transplanted human amniotic mesenchymal stromal cells into a rat uterine cavity injury model and obtained endometrial regeneration. The endometria were significantly thicker in the transplant group, with an increased number of endometrial glands and inhibition of fibrogenesis in this group. Wang et al (30) conducted a study on regeneration using intravenous or intrauterine injection of BM-MSCs in a rat model of Asherman syndrome. In both groups, endometrial fibrogenesis significantly decreased and endometrial gland cells increased in comparison with those in the non-injection group. Cervelló et al (31) injected human BM-MSCs intravenously or directly into endometria in a murine uterine cavity injury model, and observed endometrial regeneration in both group. It was proposed that endometrial regeneration was enhanced by a paracrine effect induced by transplanted cells. Alawadhi et al (32) also injected BM-MSCs intravenously into a murine uterine cavity injury model, obtaining therapeutic effects and significantly increased pregnancy rates after MSCs injection. Kilic et al (33) and Zhao et al (34) performed MSCs transplantation and conducted molecular biology studies to investigate how MSCs contribute to endometrial regeneration.

Most studies of endometrial regeneration have used animal models, but several clinical applications in humans have also been reported. Singh et al (35) showed recovered menses in patients with Asherman syndrome using mononuclear stem cells. Nagori et al (36) injected BM-MSCs and endometrial angiogenic stem cells into the uterine cavity and confirmed pregnancy by in vitro fertilization-embryo transfer. Tan et al (37) obtained recovery of endometrial thinning by injection of autologous menstrual blood-derived stromal cells in patients with Asherman syndrome, with the result of three pregnancies, with two subsequent abortions. This study had no control group, but endometria thickened from before to after treatment and embryo implantation was possible.

Uterus transplantation (UTx) is also used for AUFI. From 2012 to 2013, UTx with nine living donors was conducted in Sweden (38), using advanced technology for organ preservation and vascular anastomosis and novel immunosuppressants. In 2014, the Swedish group reported the first delivery by a patient who underwent UTx (39), and successful deliveries by nine UTx patients have been achieved by this group up to April 2018. UTx is now performed in clinical practice in many countries, and provides hope for patients with AUFI. However, further improvement of available immunosuppressants and surgical procedures for UTx are required, and there are also ethical and social concerns, including for the welfare of the child (40).

Trials of artificial uterus production can be traced back to 1954, when Greenberg connected a rat fetus, which remained bound to the umbilical cord and placenta, to an extracorporeal circulation system that served as an assumed artificial uterus, and observed changes in fetal electrolytes (41). In 1958, Westin et al tried to develop neonates using an extracorporeal circulation cycle connected to a pump and oxygenator via the umbilical artery and vein (42). Recently, in 2017, Partridge et al achieved 4-week nursing of a fetal goat in an artificial uterus (43). Recent progress with extracorporeal circulation systems supports longer fetal development in artificial uteri. However, it is still difficult to proceed from implantation to delivery in an artificial uterus.

It is difficult to make a conclusive comparison of the quality of methods to regenerate the uterus, because there were only ten articles and every method described in these studies was different to the others, even though the results were very similar and all positive. Regarding the limitations of the studies reviewed in this article, all studies were conducted only on rodents, but the anatomical features, vascular system and uterine size of rodents differ greatly from those of humans. Additionally, the duration of human pregnancy is longer, and the fetus size is relatively bigger than for rodents. These are important differences we need to consider before adopting these methods for humans. Therefore, further studies on non-human primates who have similar uterine anatomical features and vascular systems to humans are required before we progress to the clinical application of these techniques.

There has been remarkable progress in the field of regenerative medicine in the 30 years since the concept of tissue engineering was proposed; some artificial organs are already used in clinical practice. Many new techniques, such as grafts produced with cells alone and without scaffolds in cartilage regeneration (10) and in making a myocardial patch (44), and vessel production using a 3D bioprinter (45), have recently been investigated. These new technologies may be applicable to uterine regeneration.

\section{Conclusion}

Though AUFI has a major effect on the lives of patients and makes it impossible for them to have their own child, studies of uterine regenerative medicine may be delayed when compared with those on other organs, as the uterus is not a vital organ. Although there are promising results from uterine regeneration studies, to date these studies have only been conducted on animals, so the applicability of the technology requires further investigation.

\section{Acknowledgements}

Not applicable.

\section{Funding}

None of the authors received specific funding related to this article. 


\section{Availability of data and materials}

Not applicable.

\section{Authors' contributions}

YM was the main author involved in writing the article. YM, IK and AS conducted the literature search for articles on uterine regeneration. IK, KB and MY conducted the literature search for articles on regeneration medicine in other organs and on reproductive medicine. DA conceived and designed the review and suggested revisions to the article. All authors read and approved the final manuscript.

\section{Ethics approval and consent to participate}

Not applicable.

\section{Patient consent for publication}

Not applicable.

\section{Competing interests}

The authors declare that they have no competing interests.

\section{References}

1. Zegers-Hochschild F, Adamson GD, de Mouzon J, Ishihara O, Mansour R, Nygren K, Sullivan E and Vanderpoel S; International Committee for Monitoring Assisted Reproductive Technology; World Health Organization: International Committee for Monitoring Assisted Reproductive Technology (ICMART) and the World Health Organization (WHO) revised glossary of ART terminology, 2009. Fertil Steril 92: 1520-1524, 2009.

2. OppeltP,RennerSP,Kellermann A,Brucker S, HauserGA,Ludwig KS, Strissel PL, Strick R, Wallwiener D and Beckmann MW: Clinical aspects of Mayer-Rokitansky-Kuester-Hauser syndrome: Recommendations for clinical diagnosis and staging. Hum Reprod 21: 792-797, 2006.

3. Lefkowitz A, Edwards M and Balayla J: The Montreal Criteria for the Ethical Feasibility of Uterine Transplantation. Transpl Int 25: 439-447, 2012.

4. Green H, Kehinde O and Thomas J: Growth of cultured human epidermal cells into multiple epithelia suitable for grafting. Proc Natl Acad Sci USA 76: 5665-5668, 1979.

5. Langer R and Vacanti J: Advances in tissue engineering. J Pediatr Surg 51: 8-12, 2016.

6. Cima LGVJ, Vacanti JP, Vacanti C, Ingber D, Mooney D and Langer R: Tissue engineering by cell transplantation using degradable polymer substrates. J Biomech Eng 113: 143-151, 1991.

7. Thomson JA, Itskovitz-Eldor J, Shapiro SS, Waknitz MA, Swiergiel JJ, Marshall VS and Jones JM: Embryonic stem cell lines derived from human blastocysts. Science 282: 1145-1147, 1998.

8. Takahashi K, Tanabe K, Ohnuki M, Narita M, Ichisaka T, Tomoda $\mathrm{K}$ and Yamanaka S: Induction of pluripotent stem cells from adult human fibroblasts by defined factors. Cell 131: 861-872, 2007

9. Gholipourmalekabadi M, Seifalian AM, Urbanska AM, Omrani MD, Hardy JG, Madjd Z, Hashemi SM, Ghanbarian H, Brouki Milan P, Mozafari M, et al: 3D Protein-Based Bilayer Artificial Skin for the Guided Scarless Healing of Third-Degree Burn Wounds in Vivo. Biomacromolecules 19: 2409-2422, 2018

10. Ishihara K, Nakayama K, Akieda S, Matsuda S and Iwamoto Y: Simultaneous regeneration of full-thickness cartilage and subchondral bone defects in vivo using a three-dimensional scaffold-free autologous construct derived from high-density bone marrow-derived mesenchymal stem cells. J Orthop Surg Res 9: 98, 2014.
11. Dhulekar J and Simionescu A: Challenges in vascular tissue engineering for diabetic patients. Acta Biomater 70: 25-34, 2018.

12. Okamoto R, Takayama K, Akita N, Nagamoto Y, Hosokawa D, Iizuka S, Sakurai F, Suemizu H, Ohashi K and Mizuguchi H: Human iPS Cell-based Liver-like Tissue Engineering at Extrahepatic Sites in Mice as a New Cell Therapy for Hemophilia B. Cell Transplant 27: 299-309, 2018.

13. Wang Z, Lee SJ, Cheng HJ, Yoo JJ and Atala A: 3D bioprinted functional and contractile cardiac tissue constructs. Acta Biomater 70: 48-56, 2018

14. Xue A, Niu G, Chen Y, Li K, Xiao Z, Luan Y, Sun C, Xie X, Zhang D, Du X, et al: Recellularization of well-preserved decellularized kidney scaffold using adipose tissue-derived stem cells J Biomed Mater Res A 106: 805-814, 2018.

15. Yanaga $\mathrm{H}$, Imai $\mathrm{K}$, Tanaka $\mathrm{Y}$ and Yanaga $\mathrm{K}$ : Two-stage transplantation of cell-engineered autologous auricular chondrocytes to regenerate chondrofat composite tissue: Clinical application in regenerative surgery. Plast Reconstr Surg 132: 1467-1477, 2013

16. You HJ, Han SK, Lee JW and Chang H: Treatment of diabetic foot ulcers using cultured allogeneic keratinocytes - a pilot study. Wound Repair Regen 20: 491-499, 2012.

17. Shafiee A and Atala A: Tissue Engineering: Toward a New Era of Medicine. Annu Rev Med 68: 29-40, 2017.

18. Li X, Sun H, Lin N, Hou X, Wang J, Zhou B, Xu P, Xiao Z, Chen B, Dai J, et al: Regeneration of uterine horns in rats by collagen scaffolds loaded with collagen-binding human basic fibroblast growth factor. Biomaterials 32: 8172-8181, 2011

19. Santoso EG, Yoshida K, Hirota Y, Aizawa M, Yoshino O, Kishida A, Osuga Y, Saito S, Ushida T and Furukawa KS: Application of detergents or high hydrostatic pressure as decellularization processes in uterine tissues and their subsequent effects on in vivo uterine regeneration in murine models. PLoS One 9: e103201, 2014

20. Miyazaki K and Maruyama T: Partial regeneration and reconstruction of the rat uterus through recellularization of a decellularized uterine matrix. Biomaterials 35: 8791-8800, 2014.

21. Ding L, Li X, Sun H, Su J, Lin N, Péault B, Song T, Yang J, Dai J and Hu Y: Transplantation of bone marrow mesenchymal stem cells on collagen scaffolds for the functional regeneration of injured rat uterus. Biomaterials 35: 4888-4900, 2014.

22. Song T, Zhao X, Sun H, Li X, Lin N, Ding L, Dai J and $\mathrm{Hu} \mathrm{Y}$ : Regeneration of uterine horns in rats using collagen scaffolds loaded with human embryonic stem cell-derived endometrium-like cells. Tissue Eng Part A 21: 353-361, 2015.

23. Hellström M, Moreno-Moya JM, Bandstein S, Bom E, Akouri RR, Miyazaki K, Maruyama T and Brännström M: Bioengineered uterine tissue supports pregnancy in a rat model. Fertil Steril 106: 487-496.e1, 2016.

24. Hiraoka T, Hirota Y, Saito-Fujita T, Matsuo M, Egashira M, Matsumoto L, Haraguchi H, Dey SK, Furukawa KS, Fujii T, et al: STAT3 accelerates uterine epithelial regeneration in a mouse model of decellularized uterine matrix transplantation. JCI Insight 1: 1, 2016.

25. Xu L, Ding L, Wang L, Cao Y, Zhu H, Lu J, Li X, Song T, Hu Y and Dai J: Umbilical cord-derived mesenchymal stem cells on scaffolds facilitate collagen degradation via upregulation of MMP-9 in rat uterine scars. Stem Cell Res Ther 8: 84, 2017.

26. Campbell GR, Turnbull G, Xiang L, Haines M, Armstrong S, Rolfe BE and Campbell JH: The peritoneal cavity as a bioreactor for tissue engineering visceral organs: Bladder, uterus and vas deferens. J Tissue Eng Regen Med 2: 50-60, 2008.

27. Lin N, Li X, Song T, Wang J, Meng K, Yang J, Hou X, Dai J and $\mathrm{Hu} \mathrm{Y}$ : The effect of collagen-binding vascular endothelial growth factor on the remodeling of scarred rat uterus following full-thickness injury. Biomaterials 33: 1801-1807, 2012.

28. Gil-Sanchis C, Cervello I, Khurana S, Faus A, Verfaillie C and Simon C: Contribution of different bone marrow-derived cell types in endometrial regeneration using an irradiated murine model. Fertil Steril 103: 1596-1605.e1, 2015.

29. Gan L, Duan H, Xu Q, Tang YQ, Li JJ, Sun FQ and Wang S: Human amniotic mesenchymal stromal cell transplantation improves endometrial regeneration in rodent models of intrauterine adhesions. Cytotherapy 19: 603-616, 2017.

30. Wang J, Ju B, Pan C, Gu Y, Zhang Y, Sun L, Zhang B and Zhang Y: Application of Bone Marrow-Derived Mesenchymal Stem Cells in the Treatment of Intrauterine Adhesions in Rats. Cell Physiol Biochem 39: 1553-1560, 2016. 
31. Cervelló I, Gil-Sanchis C, Santamaría X, Cabanillas S, Díaz A Faus A, Pellicer A and Simón C: Human CD133(+) bone marrow-derived stem cells promote endometrial proliferation in a murine model of Asherman syndrome. Fertil Steril 104: 1552-1560.e1-3, 2015.

32. Alawadhi F, Du H, Cakmak $\mathrm{H}$ and Taylor HS: Bone Marrow-Derived Stem Cell (BMDSC) transplantation improves fertility in a murine model of Asherman's syndrome. PLoS One 9: e96662, 2014.

33. Kilic S, Yuksel B, Pinarli F, Albayrak A, Boztok B and Delibasi T: Effect of stem cell application on Asherman syndrome, an experimental rat model. J Assist Reprod Genet 31: 975-982, 2014

34. Zhao J, Zhang Q, Wang Y and Li Y: Uterine infusion with bone marrow mesenchymal stem cells improves endometrium thickness in a rat model of thin endometrium. Reprod Sci 22: 181-188, 2015.

35. Singh N, Mohanty S, Seth T, Shankar M, Bhaskaran S and Dharmendra S: Autologous stem cell transplantation in refractory Asherman's syndrome: A novel cell based therapy. J Hum Reprod Sci 7: 93-98, 2014.

36. Nagori CB, Panchal SY and Patel H: Endometrial regeneration using autologous adult stem cells followed by conception by in vitro fertilization in a patient of severe Asherman's syndrome. J Hum Reprod Sci 4: 43-48, 2011.

37. Tan J, Li P, Wang Q, Li Y, Li X, Zhao D, Xu X and Kong L: Autologous menstrual blood-derived stromal cells transplantation for severe Asherman's syndrome. Hum Reprod 31: 2723-2729, 2016.

38. Brännström M, Johannesson L, Dahm-Kähler P, Enskog A, Mölne J, Kvarnström N, Diaz-Garcia C, Hanafy A, Lundmark C, Marcickiewicz J, et al: First clinical uterus transplantation trial: A six-month report. Fertil Steril 101: 1228-1236, 2014.
39. Brännström $M$, Johannesson L, Bokström $H$, Kvarnström $N$, Mölne J, Dahm-Kähler P, Enskog A, Milenkovic M, Ekberg J, Diaz-Garcia C, et al: Livebirth after uterus transplantation. Lancet 385: 607-616, 2015.

40. Suganuma N, Hayashi A, Kisu I, Banno K, Hara H and Mihara M: Uterus transplantation: Toward clinical application in Japan. Reprod Med Biol 16: 305-313, 2017.

41. Greenberg EM: The artificial uterus and the passage of radioactive sodium through hemochorial mammalian placenta extra utero. Bull N Y Acad Med 30: 713-714, 1954.

42. Westin B, Nyberg R and Enhorning G: A technique for perfusion of the previable human fetus. Acta Paediatr (Stockh) 47: 339-349, 1958.

43. Partridge EA, Davey MG, Hornick MA, McGovern PE, Mejaddam AY, Vrecenak JD, Mesas-Burgos C, Olive A, Caskey RC, Weiland TR, et al: An extra-uterine system to physiologically support the extreme premature lamb. Nat Commun 8: 15112,2017

44. Noguchi R, Nakayama K, Itoh M, Kamohara K, Furukawa K, Oyama J, Node K and Morita S: Development of a three-dimensional pre-vascularized scaffold-free contractile cardiac patch for treating heart disease. J Heart Lung Transplant 35: 137-145, 2016.

45. Itoh M, Nakayama K, Noguchi R, Kamohara K, Furukawa K, Uchihashi K, Toda S, Oyama J, Node K and Morita S: Scaffold-free tubular tissues created by a Bio-3D printer undergo remodeling and endothelialization when implanted in rat aortae. PLoS One 10: e0136681, 2015. 